\title{
Assessing Potato Cultivar Sensitivity to Tuber Necrosis Caused by Potato mop-top virus
}

S. K. R. Yellareddygari, Department of Plant Pathology, North Dakota State University, Fargo, ND, 58105; Jonathan L. Whitworth, USDA-ARS, Aberdeen, Idaho, 83210; and Neil C. Gudmestad, ${ }^{\dagger}$ Department of Plant Pathology, North Dakota State University, Fargo, ND, 58105

\begin{abstract}
Potato mop-top virus (PMTV) causes mop top disease in potato. This disease can result in a decline in tuber quality causing economic losses to growers due to the production of necrotic lesions and discolored tissue in infected tubers. Due to the soilborne nature of PMTV, identifying and developing host resistance against the virus is considered the best disease management option. Very little is known about the sensitivity of U.S. potato cultivars to PMTV-induced tuber necrosis. The current study is aimed at investigating the sensitivity of a large number of potato cultivars to PMTV-induced tuber necrosis. Sixty-three cultivars representing all market-types were evaluated in North Dakota over a 2-year period for virus-induced tuber necrosis incidence and severity. PMTV-induced

tuber necrosis $(P<0.0001)$ and severity $(P<0.0001)$ were significantly different among cultivars. Cultivars were categorized into sensitive, insensitive, and moderately sensitive/insensitive groups based on the virus-tuber induced necrosis data from both years. Based on data from ND trials, six cultivars (Red Endeavor, Viking, Dakota Jewel, Dark Red Norland, Nicolet, and Modoc) were rated as sensitive and 43 were rated as insensitive to PMTV-induced tuber necrosis. Four cultivars, including Bannock Russet, Gemstar Russet, Lelah, and Waneta showed zero PMTV incidence over 2 years. These results will help growers in making individual or coordinated decisions for the management of PMTV-induced tuber necrosis under field and storage conditions.
\end{abstract}

Potato mop top disease, caused by Potato mop-top virus (PMTV), is an important disease in many U.S. potato production regions. PMTV is vectored by the soilborne plasmodiophorid Spongospora subterranea (Wallroth) Lagerheim (Tenorio et al. 2006). PMTV remains infective in soil for prolonged periods by surviving in resting spores of S. subterranea (Calvert 1968; Jones and Harrison 1969; Kirk 2008; Santala et al. 2010). PMTV is an important pathogen of potato and can cause serious economic losses in sensitive cultivars (Domfeh et al. 2015b; Harrison and Jones 1970; Sandgren et al. 2002). The probable geographic origin of PMTV is the Andean region of South America and it is now present in many potato growing countries in North America, Europe, and Asia (Beuch et al. 2014; Hu et al. 2013; Santala et al. 2010; Xu et al. 2004). PMTV is becoming an emerging threat in U.S., where it was first reported in Maine (Lambert et al. 2003) and later in Colorado, Idaho, New Mexico, North Dakota, and Washington (David et al. 2010; Mallik and Gudmestad 2015; Whitworth and Crosslin 2013).

Characteristic symptoms of PMTV include internal dark-colored arcs or concentric rings and on occasion, external tuber and foliar symptoms may also appear (Calvert and Harrison 1966; Domfeh and Gudmestad 2016; Harrison and Jones 1970). PMTV symptoms are very similar to Tobacco rattle virus (TRV) caused corky ring spot disease symptoms (Beuch et al. 2014; Mumford et al. 2000). Secondary PMTV infections may cause dwarfed appearance of the plant due to shortening of internodes (Calvert 1968; Domfeh et al. 2015a). Prevailing environmental conditions can strongly affect foliar symptoms and transmission of PMTV by vector (Calvert 1968; Carnegie et al. 2012; Cooper and Harrison 1973; Domfeh et al. 2015b; Harrison 1974; Sandgren et al. 2002). Tuber necrosis among potato cultivars and across years varied due to varying soil moisture and temperatures (Domfeh et al. 2015b). Although PMTV has no direct impact on yield loss, it may incur quality loss, which can lead to rejection of tubers intended for the fresh market and processing industry (Mumford et al. 2000).

${ }^{\dagger}$ Corresponding author: Neil C. Gudmestad;

E-mail: neil.gudmestad@ndsu.edu

Funding: USDA-NIFA-SCRI grant number 2014-51181-22373.

Accepted for publication 6 January 2018.

() 2018 The American Phytopathological Society
Identifying natural resistance in potato genotypes is the best method for managing PMTV (Domfeh et al. 2015a). Unfortunately, the breeding of genetic resistance to a virus in an autotetraploid potato cultivar can be a lengthy process. In the absence of genetic resistance, determining the sensitivity of potato cultivars to the expression of tuber necrosis incited by PMTV infection may be a viable alternative to reduce the economic impact of this virus. Several studies in Europe have evaluated potato cultivars for sensitivity to PMTVinduced tuber necrosis (Calvert 1968; Carnegie et al. 2009, 2010a; Kurppa 1989; Nielsen and Engsbro 1992; Nielsen and Molgaard 1997; Sandgren 1995; Sandgren et al. 2002). Comprehensive evaluation of potato cultivars grown in the U.S. for sensitivity to PMTVinduced tuber necrosis is limited. As per the information of The European Cultivated Potato Database, zero cultivars were screened for PMTV-induced tuber necrosis in Europe. A recent study evaluated 13 U.S. potato cultivars for sensitivity to PMTV-induced tuber necrosis (Domfeh et al. 2015a). However, no studies have been conducted to assess currently grown potato cultivars belonging to all market types in the U.S. for sensitivity to PMTV-induced tuber necrosis. Therefore, the objective of the current study is to evaluate sensitivity of 63 commonly grown U.S. potato cultivars for their reaction to PMTV-tuber induced necrosis.

\section{Materials and Methods}

Two field trials (2015 and 2016) were established near McCanna, ND, to screen cultivars for sensitivity to PMTV-induced tuber necrosis in a randomized complete block design (RCBD) with three replications. This trial location has been demonstrated to be infested with PMTV viruliferous $S$. subterranea and has been used to previously evaluate the sensitivity of potato cultivars and advanced potato breeding selections (Domfeh et al. 2015a, b). A total of 63 cultivars were planted in 2015 (Table 1). In 2016, 60 potato cultivars were planted for PMTV trial and three cultivars (POR06V12-3, Lelah, and Rio Colorado) were omitted due to lack of seed material. Each replication consisted of 15 tubers per cultivar planted at $12 \mathrm{~cm}$ soil depth and $0.3 \mathrm{~m}$ spacing. Potato vines were desiccated by flailing using a mechanical vine beater and harvested tubers were transported to storage facility located at NDSU for postharvest assessment. Approximately half of the harvested tubers were stored at room temperature and were assessed for respective necrosis symptoms (first assessment). The remaining tubers were maintained at storage conditions of $10^{\circ} \mathrm{C}$ and $85 \%$ humidity until their evaluation for PMTVinduced necrosis (second assessment). 
In total, two postharvest visual assessments of PMTV-induced necrosis in tubers were performed using previously published protocols (Domfeh et al. 2015a, b; Nielsen and Molgaard 1997; Yellareddygari et al. 2017). The first and second tuber necrosis assessments were performed at 20 and 119 and 60 and 156 days postharvest (DPH) for the 2015 and 2016 trials, respectively. For each evaluation, a sample consisting of 25 random tubers per cultivar from each replication were evaluated. Tubers were washed, cut lengthwise into $1-\mathrm{cm}$ thick slices using a mandolin slicer (Jaccard Corporation, NY), and disease incidence and severity were evaluated. Virus severity was measured as a proportion of tuber affected with a necrosis symptom. First, the number of slices $(x)$ showing virus symptoms was determined. A tuber slice with the most severe internal necrosis was visually identified and covered with a transparent sheet consisting of 1-cm squares. The number of squares overlapping necrosis symptoms was recorded $(y)$. Virus incidence was calculated by dividing the number of tubers with virus induced necrosis symptoms with total number of tubers assessed. Disease severity index for virus was calculated as $x \times y$ and expressing the values between 0 and 1 , where 1 indicates that whole tuber has necrosis and 0 indicates absence of necrosis.

Detection of PMTV in tubers. Approximately 30 random symptomatic tubers during the second assessment were assayed to confirm PMTV using reverse-transcription (RT)-PCR (Domfeh et al. 2015a, b). A sterilized scalpel was used to remove infected tissue from slices of potato tubers, which were immediately crushed in liquid nitrogen and stored at $-80^{\circ} \mathrm{C}$. TRIzol reagent (Life Technologies, Grand Island, $\mathrm{NY}$ ) was used to extract total RNA as per the manufacturer's instructions. Air dried RNA pellets were dissolved in $100 \mu \mathrm{l}$ of RNase-free water. PMTV was detected using previously published protocols (Domfeh et al. 2015a; Nakayama et al. 2010). The PCR reaction mixture for PMTV detection contained $2 \mu \mathrm{l}$ of $2.5 \mathrm{mM}$ dNTPs, $0.5 \mu \mathrm{l}$ each of $10 \mu \mathrm{M}$ forward (PMTV-(9P; 5'-GCTTGATCCAGAAGTCATT AAGG-3') and reverse (PMTV-9M; 5'-CCTGGAAGCACCAATAC TTAACG-3') (Nakayama et al. 2010). Furthermore, another 20 random asymptomatic tuber samples per pathogen trial and per year were tested using multiplex real-time fluorescent RT-PCR according to previously published protocol (Mumford et al. 2000). Multiplex assay (duplex) was used to test whether the necrosis was actually caused by PMTV and not due to TRV infection.

Statistical analysis. Statistical analysis software (SAS version 9.3) was used for the analysis of variance (ANOVA) of virus-induced tuber necrosis incidence and severity data. In both pathogen trials, homogeneity of variance test was significant for virus-induced necrosis incidence $(P<0.0001)$ and severity $(P<0.001)$ and data from experimental tuber necrosis assessments were analyzed separately.
Analyses were conducted separately for years due to the differences in number of cultivars. A paired $t$ test was performed to determine whether the means (virus-induced tuber necrosis) of two groups (two assessments and two years) were statistically different from each other. Pearson correlation coefficient was used to study the relationship between virus-induced tuber necrosis vs. severity index and first assessment vs. second assessment. Additionally, virus-induced tuber necrosis incidence assessment from both assessments and years were used for cultivar sensitivity ranking/grouping (Domfeh et al. 2015a). Potato cultivars were categorized as insensitive (overall incidence $<5 \%$ ), moderately insensitive (overall incidence $>5$ to $10 \%$ ), moderately sensitive (overall incidence $>10$ to $15 \%$ ), and sensitive (overall incidence $>15 \%$ ) groups.

\section{Results}

PMTV field trial, 2015. The incidence of PMTV-induced tuber necrosis was low during the first assessment and few cultivars were affected by the virus. Of the 63 cultivars tested, only 10 exhibited PMTV necrosis symptoms in tubers. Significant differences were found among cultivars for PMTV-induced tuber necrosis incidence $(P<0.0001)$ and severity $(P<0.0003)$. PMTV tuber necrosis incidence ranged from 0 to $21.9 \%$ and severity ranged from 0 to 0.13 (Table 2). Tubers of cultivars Red Endeavor and Nicolet exhibited more than $10 \%$ disease incidence (Table 2).

Table 2. Mean incidence and severity of Potato mop-top virus (PMTV) induced tuber necrosis in 63 cultivars, 2015 ND trial (first assessment) ${ }^{\mathrm{y}}$

\begin{tabular}{lcc}
\hline Cultivar & Incidence (\%) & Severity index \\
\hline Red Endeavor & $21.9 \mathrm{a}$ & $0.13 \mathrm{a}$ \\
Nicolet & $12.2 \mathrm{~b}$ & $0.08 \mathrm{bc}$ \\
Snowden & $3.6 \mathrm{c}$ & $0.11 \mathrm{ab}$ \\
Russet Norkotah 278 & $3.2 \mathrm{c}$ & $0.07 \mathrm{bc}$ \\
Modoc & $2.6 \mathrm{c}$ & $0.03 \mathrm{~cd}$ \\
Dakota Jewel & $2.6 \mathrm{c}$ & $0.01 \mathrm{~d}$ \\
Premier Russet & $1.5 \mathrm{c}$ & $0.01 \mathrm{~d}$ \\
Snowbird & $1.3 \mathrm{c}$ & $0.01 \mathrm{~d}$ \\
Dakota Ruby & $1.2 \mathrm{c}$ & $0.01 \mathrm{~d}$ \\
Lelah & $0.9 \mathrm{c}$ & $0.02 \mathrm{~d}$ \\
Others & $0.0 \mathrm{c}$ & $0.00 \mathrm{~d}$ \\
LSD $_{\mathbf{0 . 0 5}}^{\mathrm{z}}$ & $\mathbf{3 . 7}$ & $\mathbf{0 . 0 5}$ \\
\hline
\end{tabular}

${ }^{y}$ Means separated by the same letter are not significantly different according to Fisher's protected least significant difference $(\alpha<0.05)$.

${ }^{\mathrm{z}}$ Cultivars with no apparent PMTV-induced tuber necrosis symptoms and not listed in the table.

Table 1. Potato cultivars, sorted by market type, evaluated for tuber necrosis induced by Potato mop-top virus ${ }^{\mathrm{z}}$

\begin{tabular}{|c|c|c|c|c|c|}
\hline Russet (French fry/processing) & White (chipping) & Red & Russet tablestock & Specialty & Yellow \\
\hline Alpine Russet & Atlantic & Chieftain & Goldrush & Austrian Crescent & Bintje \\
\hline Alturas & Chipeta & Ciklamen & Russet Norkotah & Desiree & Gala \\
\hline POR06V12-3 & Dakota Crisp & Colorado Rose & Russet Norkotah 278 & French Fingerling & Oneida Gold \\
\hline Bannock Russet & Dakota Pearl & Dakota Jewel & Russet Norkotah 296 & Huckleberry Gold & Yukon Gold \\
\hline Centennial Russet & Kennebec & Dakota Ruby & Russet Norkotah CO3 & Russian Banana & \\
\hline Classic Russet & Lamoka & Dark Red Norland & Russet Norkotah CO8 & Snowden & \\
\hline Clearwater Russet & Lelah & Modoc & & & \\
\hline Dakota Russet & Marcy & Red Endeavor & & & \\
\hline Dakota Trailblazer & Mega Chip & Red Gold & & & \\
\hline Freedom Russet & Nicolet & Red LaSoda & & & \\
\hline Gemstar Russet & Pike & Red Norland & & & \\
\hline Premier Russet & Pinnacle & Red Thumb & & & \\
\hline Ranger Russet & Snowbird & Rio Colorado & & & \\
\hline Russet Burbank & Superior & & & & \\
\hline Sage Russet & Waneta & & & & \\
\hline \multicolumn{6}{|l|}{ Silverton Russet } \\
\hline \multicolumn{6}{|l|}{ Teton Russet } \\
\hline Umatilla Russet & & & & & \\
\hline
\end{tabular}

${ }^{\mathrm{z}}$ Potato cultivars PORO6V12-3, Lelah, and Rio Colorado were included only in the 2015 McCanna trial, ND. 
The incidence of PMTV-induced tuber necrosis increased among cultivars during the second evaluation. The number of cultivars affected by PMTV tuber necrosis also increased (Table 3). PMTVinduced tuber necrosis incidence $(P<0.0001)$ and severity $(P<$ $0.0001)$ were significantly different among cultivars. PMTVinduced tuber necrosis ranged from 0 to $33.4 \%$, while severity ranged from 0 to 0.49 (Table 3). Nicolet, followed by Red Endeavor and Snowden, had the highest incidence of $(>30 \%)$ PMTV-induced necrosis in tubers.
PMTV field trial, 2016. In the first assessment of PMTV-induced tuber necrosis, the number of cultivars with PMTV-induced tuber necrosis was higher in 2016 compared with 2015 (36 cultivars with symptoms). PMTV-induced tuber necrosis $(P<0.0001)$ and severity $(P<0.0001)$ indices were significantly different among the cultivars. Cultivars Red Endeavor, Dakota Jewel, and Dark Red Norland had the highest PMTV-induced tuber necrosis incidence (Table 4).

Significant differences in the incidence $(P<0.0001)$ and severity $(P<0.0001)$ of PMTV-induced tuber necrosis were detected among

Table 3. Mean incidence and severity of Potato mop-top virus (PMTV)-induced tuber necrosis in 63 cultivars, 2015 ND trial (second assessment)

\begin{tabular}{|c|c|c|c|c|c|}
\hline Cultivar & Incidence (\%) & Severity index & Cultivar & Incidence (\%) & Severity index \\
\hline Nicolet & $33.4 \mathrm{a}$ & 0.18 cdef & Pinnacle & $3.8 \mathrm{fgh}$ & $0.05 \mathrm{gf}$ \\
\hline Red Endeavor & $31.6 \mathrm{a}$ & $0.36 \mathrm{ab}$ & Red Gold & $3.6 \mathrm{fgh}$ & $0.01 \mathrm{~g}$ \\
\hline Snowden & $30.4 \mathrm{a}$ & 0.21 bcde & Alpine Russet & $3.5 \mathrm{fgh}$ & $0.03 \mathrm{~g}$ \\
\hline Modoc & $21.6 \mathrm{~b}$ & $0.29 \mathrm{bc}$ & Silverton Russet & $2.9 \mathrm{gh}$ & $0.02 \mathrm{~g}$ \\
\hline Lelah & $16.4 \mathrm{bc}$ & $0.49 \mathrm{a}$ & Teton Russet & $2.7 \mathrm{gh}$ & $0.02 \mathrm{~g}$ \\
\hline Waneta & $16.3 \mathrm{bcd}$ & 0.11 defg & Clearwater Russet & $2.5 \mathrm{gh}$ & $0.04 \mathrm{gf}$ \\
\hline French Fingerling & 13.7 bcde & $0.24 \mathrm{bcd}$ & Russet Norkotah 278 & $2.4 \mathrm{gh}$ & 0.13 defg \\
\hline Dark Red Norland & $11.7 \mathrm{cdef}$ & $0.11 \mathrm{~g}$ & Dakota Ruby & $2.4 \mathrm{gh}$ & 0.21 bcde \\
\hline Dakota Crisp & 9.6 cdefg & 0.14 cdefg & Superior & $2.3 \mathrm{gh}$ & 0.10 defg \\
\hline Chieftain & 8.0 defgh & $0.09 \mathrm{efg}$ & Red Norland & $2.2 \mathrm{gh}$ & $0.05 \mathrm{gf}$ \\
\hline Kennebec & 7.9 defgh & $0.02 \mathrm{~g}$ & Russet Norkotah CO3 & $2.2 \mathrm{gh}$ & $0.02 \mathrm{~g}$ \\
\hline Dakota Jewel & $7.8 \mathrm{efgh}$ & $0.10 \mathrm{~g}$ & Viking & $1.9 \mathrm{gh}$ & $0.01 \mathrm{~g}$ \\
\hline Pike & $7.2 \mathrm{efgh}$ & $0.07 \mathrm{efg}$ & Lamoka & $1.5 \mathrm{gh}$ & $0.02 \mathrm{~g}$ \\
\hline Gala & 6.9 efgh & $0.08 \mathrm{efg}$ & Mega Chip & $1.5 \mathrm{gh}$ & $0.01 \mathrm{~g}$ \\
\hline Rio Colorado & 6.9 efgh & $0.07 \mathrm{efg}$ & Austrian Crescent & $1.4 \mathrm{gh}$ & $0.24 \mathrm{bcd}$ \\
\hline Red LaSoda & 6.5 efgh & $0.05 \mathrm{gf}$ & Dakota Pearl & $1.4 \mathrm{gh}$ & 0.01 defg \\
\hline Ciklamen & $5.8 \mathrm{efgh}$ & 0.14 cdefg & Chipeta & $1.3 \mathrm{gh}$ & $0.01 \mathrm{~g}$ \\
\hline Russet Norkotah CO8 & $5.6 \mathrm{efgh}$ & $0.03 \mathrm{~g}$ & Ranger Russet & $1.3 \mathrm{gh}$ & $0.01 \mathrm{~g}$ \\
\hline Russet Burbank & $5.2 \mathrm{fgh}$ & $0.07 \mathrm{efg}$ & Atlantic & $1.2 \mathrm{gh}$ & $0.01 \mathrm{~g}$ \\
\hline Desiree & $4.9 \mathrm{fgh}$ & $0.06 \mathrm{efg}$ & Oneida Gold & $1.1 \mathrm{~h}$ & $0.01 \mathrm{~g}$ \\
\hline Red Thumb & $4.8 \mathrm{fgh}$ & $0.08 \mathrm{efg}$ & Bintje & $0.9 \mathrm{~h}$ & $0.01 \mathrm{~g}$ \\
\hline Colorado Rose & $4.6 \mathrm{fgh}$ & $0.02 \mathrm{~g}$ & Huckleberry Gold & $0.9 \mathrm{~h}$ & 0.11 defg \\
\hline Marcy & $4.5 \mathrm{fgh}$ & $0.02 \mathrm{~g}$ & Russian Banana & $0.4 \mathrm{~h}$ & $0.02 \mathrm{~g}$ \\
\hline Snowbird & $3.9 \mathrm{fgh}$ & $0.04 \mathrm{gf}$ & Others ${ }^{\mathrm{z}}$ & $0.0 \mathrm{~h}$ & $0.00 \mathrm{~g}$ \\
\hline $\mathbf{L S D}_{0.05}$ & & & & 8.4 & 0.15 \\
\hline
\end{tabular}

y Means separated by the same letter are not significantly different according to Fisher's protected least significant difference $(\alpha<0.05)$.

${ }^{\text {z }}$ Cultivars (Alturas, POR06V12-3, Bannock Russet, Centennial Russet, Classic Russet, Dakota Russet, Dakota Trailblazer, Freedom Russet, Gemstar Russet, Goldrush, Premier Russet, Russet Norkotah, Russet Norkotah 296, Sage Russet, Umatilla Russet, and Yukon Gold) with no apparent PMTV-induced tuber necrosis symptoms and not listed in the table.

Table 4. Mean incidence and severity of Potato mop-top virus (PMTV)-induced tuber necrosis in 60 cultivars, 2016 ND trial (first assessment) ${ }^{\mathrm{y}}$

\begin{tabular}{|c|c|c|c|c|c|}
\hline Cultivar & Incidence (\%) & Severity index & Cultivar & Incidence (\%) & Severity index \\
\hline Red Endeavor & $26.9 \mathrm{a}$ & $0.46 \mathrm{a}$ & Dakota Trailblazer & $1.9 \mathrm{hi}$ & $0.03 \mathrm{fgh}$ \\
\hline Dakota Jewel & $23.9 \mathrm{ab}$ & $0.28 \mathrm{~b}$ & Huckleberry Gold & $1.7 \mathrm{i}$ & 0.08 defg \\
\hline Dark Red Norland & $21.4 \mathrm{abc}$ & $0.17 \mathrm{c}$ & Red Thumb & $1.7 \mathrm{i}$ & $0.01 \mathrm{fgh}$ \\
\hline Red Norland & $18.9 \mathrm{bcd}$ & $0.35 \mathrm{~b}$ & Colorado Rose & $1.6 \mathrm{i}$ & $0.01 \mathrm{fhg}$ \\
\hline Chieftain & $18.7 \mathrm{bcd}$ & $0.19 \mathrm{c}$ & Pike & $1.6 \mathrm{i}$ & $0.02 \mathrm{fgh}$ \\
\hline Viking & $16.0 \mathrm{cde}$ & $0.19 \mathrm{c}$ & Russet Norkotah CO8 & $1.5 \mathrm{i}$ & $0.01 \mathrm{fgh}$ \\
\hline Modoc & 14.2 def & 0.12 cde & Premier Russet & $0.8 \mathrm{i}$ & $0.01 \mathrm{fgh}$ \\
\hline Nicolet & $11.6 \mathrm{efg}$ & $0.13 \mathrm{~cd}$ & Sage Russet & $0.8 \mathrm{i}$ & 0.06 defgh \\
\hline French Fingerling & $8.3 \mathrm{fgh}$ & 0.08 def & Russet Norkotah 296 & $0.8 \mathrm{i}$ & $0.02 \mathrm{fgh}$ \\
\hline Yukon Gold & $5.8 \mathrm{ghi}$ & $0.03 \mathrm{fgh}$ & Superior & $0.8 \mathrm{i}$ & $0.00 \mathrm{gh}$ \\
\hline Snowbird & $5.8 \mathrm{ghi}$ & $0.03 \mathrm{fgh}$ & Alpine Russet & $0.8 \mathrm{i}$ & $0.05 \mathrm{fgh}$ \\
\hline Dakota Pearl & $4.1 \mathrm{hi}$ & 0.07 defgh & Red LaSoda & $0.8 \mathrm{i}$ & $0.01 \mathrm{fgh}$ \\
\hline Kennebec & $3.7 \mathrm{hi}$ & $0.02 \mathrm{fgh}$ & Russet Norkotah 278 & $0.8 \mathrm{i}$ & $0.00 \mathrm{gh}$ \\
\hline Alturas & $3.5 \mathrm{hi}$ & $0.01 \mathrm{fgh}$ & Silverton Russet & $0.8 \mathrm{i}$ & $0.01 \mathrm{fgh}$ \\
\hline Clearwater Russet & $3.4 \mathrm{hi}$ & $0.02 \mathrm{fgh}$ & Dakota Crisp & $0.8 \mathrm{i}$ & 0.05 efgh \\
\hline Dakota Russet & $3.3 \mathrm{hi}$ & $0.02 \mathrm{fgh}$ & Lamoka & $0.8 \mathrm{i}$ & $0.01 \mathrm{fgh}$ \\
\hline Dakota Ruby & $3.2 \mathrm{hi}$ & $0.03 \mathrm{fgh}$ & Freedom Russet & $0.6 \mathrm{i}$ & $0.00 \mathrm{~h}$ \\
\hline Marcy & $2.6 \mathrm{hi}$ & $0.01 \mathrm{fgh}$ & Others ${ }^{\mathrm{z}}$ & $0.0 \mathrm{i}$ & $0.00 \mathrm{~h}$ \\
\hline Snowden & $2.5 \mathrm{hi}$ & 0.06 defgh & & & \\
\hline $\mathbf{L S D}_{\mathbf{0 . 0 5}}$ & & & & 6.4 & 0.08 \\
\hline
\end{tabular}

y Means separated by the same letter are not significantly different according to Fisher's protected least significant difference $(\alpha<0.05)$.

${ }^{z}$ Cultivars (Atlantic, Waneta, Austrian Crescent, Bannock Russet, Bintje, Centennial Russet, Chipeta, Ciklamen, Classic Russet, Desiree, Gala, Gemstar Russet, Goldrush, Mega Chip, Oneida Gold, Pinnocle, Ranger Russet, Red Gold, Russet Burbank, Russet Norkotah, Russet Norkotah CO3, Russian Banana, Teton Russet, and Umatilla Russet) with no apparent PMTV-induced tuber necrosis symptoms and not listed in the table. 
potato cultivars during the second assessment of tubers. PMTVinduced necrosis incidence ranged from 0 to $65 \%$ (Table 5). PMTV tuber necrosis severity ranged from 0 to 0.38 . Similar to first assessment, Dakota Jewel, Dark Red Norland, and Red Endeavor cultivars showed high incidence ( $>45 \%)$. RT-PCR and multiplex assay results from randomly selected tubers confirmed PMTV and detected only PMTV, respectively.

Based on the two years of field trials for the reaction of cultivars to PMTV-induced tuber necrosis, cultivars Red Endeavor, Viking, Dakota Jewel, Dark Red Norland, Nicolet, and Modoc were ranked as sensitive (Table 6). Furthermore, 43 cultivars were ranked as insensitive and all other cultivars were ranked as either moderately sensitive or moderately insensitive to PMTV-induced tuber necrosis.

For the 2015 PMTV trial, paired $t$ test results demonstrated that incidence of PMTV-induced tuber necrosis $(0.81 \%)$ in the first assessment was lower than second assessment $(5.1 \%)$. The mean difference between two assessments was $-4.3 \%$ and significant $(P<0.0001)$, an indication that there was a statistically significant increase in the incidence of PMTV-induced tuber necrosis in the second assessment. Similar results were observed in the incidence of PMTV-induced tuber necrosis in 2016 trial for the first assessment. The incidence of PMTV tuber necrosis was lower (3.61\%) compared with the second assessment $(13.73 \%)$, with a significant $(P<0.0001)$ increase of $10.13 \%$. Across years, PMTV-induced tuber necrosis was higher in the 2016 trial (8.67\%) compared with the 2015 trial (2.89\%), demonstrating that necrosis increased $(5.78 \%)$ significantly $(P<0.0001)$ in the 2016 trial.

Pearson correlation studies. Pearson correlation coefficient (first assessment) between PMTV-induced necrosis incidence and severity was very strong and significant during $2015(r=0.81, P<0.0001)$ and $2016(r=0.83 ; P<0.0001)$ trials. In the second assessment, the correlation coefficient between PMTV-induced necrosis incidence and severity was strong and significant for $2015(r=0.60, P<0.0001)$ and
2016 ( $r=0.81, P<0.0001)$ trials. Furthermore, the correlation between PMTV-induced tuber necrosis incidence and severity among cultivars across years was strong and significant $(r=0.72, P<0.0001)$. The correlation of PMTV-induced tuber necrosis incidence between first and second assessment was also high, $0.71(P=0.004)$ and 0.79 $(P<0.0001)$ for 2015 and 2016 trials, respectively. The correlation between years for first assessment of PMTV-tuber-necrosis incidence was significant $(r=0.54, P<0.0001)$. A significant correlation between years for the second assessment of PMTV-induced tuber necrosis was also observed $(r=0.46, P<0.0001)$.

\section{Discussion}

In the future, PMTV-induced tuber necrosis losses may be reduced by using cultivars resistant to this virus. Current cultivars with genetic resistance and improved breeder clones are sources of host resistance in potato (Solomon-Blackburn and Barker 1999). However, the lack of information regarding potato cultivar susceptibility levels can hinder the development of virus resistance in breeding programs. Cultivar screening results from studies such as the one detailed here will help in identifying resistance genetic resource material for future breeding programs. In the interim, the results from this study will help growers with PMTV-infested fields make cultivar planting choices based on the targeted potato market by avoiding those cultivars highly sensitive to the tuber necrosis phase of PMTV.

PMTV-induced necrosis incidence and severity index at both assessments varied among the cultivars, which is similar to previous cultivar screening studies (Domfeh et al. 2015a, b). It appears that several factors may influence the PMTV-tuber necrosis levels in cultivars after harvest. PMTV-induced tuber necrosis increased over postharvest storage duration. The mean PMTV-induced tuber necrosis incidence $(\%)$ over postharvest storage increased by 4.27 and 10.12 in 2015 and 2016 trials, respectively. It should be noted that timing of PMTV-induced necrosis assessment after harvest/prior to

Table 5. Mean incidence and severity of Potato mop-top virus (PMTV)-induced tuber necrosis in 60 cultivars, 2016 ND trial (second assessment) ${ }^{\mathrm{y}}$

\begin{tabular}{|c|c|c|c|c|c|}
\hline Cultivar & Incidence (\%) & Severity index & Cultivar & Incidence (\%) & Severity index \\
\hline Dakota Jewel & $65.3 \mathrm{a}$ & $0.39 \mathrm{a}$ & Dakota Russet & 9.3 hijklmno & 0.17 cdefg \\
\hline Dark Red Norland & $53.3 \mathrm{ab}$ & $0.30 \mathrm{ab}$ & Freedom Russet & 9.3 hijklmno & 0.04 klmno \\
\hline Red Endeavor & $46.7 \mathrm{bc}$ & $0.26 \mathrm{bcd}$ & Russet Norkotah 296 & 9.3 hijklmno & 0.02 no \\
\hline Dakota Pearl & $41.3 \mathrm{bcd}$ & $0.27 \mathrm{bc}$ & Silverton Russet & 9.3 hijklmno & 0.06 ijklmno \\
\hline Viking & $38.7 \mathrm{~cd}$ & 0.19 cdef & Colorado Rose & 8.0 ijklmno & 0.05 jklmno \\
\hline Modoc & $37.3 \mathrm{~cd}$ & 0.23 bcde & Snowbird & $8.0 \mathrm{ijklmno}$ & 0.04 klmno \\
\hline Chieftain & $32.0 \mathrm{de}$ & 0.17 defg & Russet Norkotah $\mathrm{CO} 3$ & 6.7 jklmno & 0.01 no \\
\hline Dakota Crisp & $29.3 \mathrm{def}$ & 0.19 cdef & Umatilla Russet & $6.7 \mathrm{jklmno}$ & 0.04 lmno \\
\hline Gala & $29.3 \mathrm{def}$ & 0.08 ghijklmno & Atlantic & $5.3 \mathrm{klmno}$ & 0.01 no \\
\hline Nicolet & $24.0 \mathrm{efg}$ & 0.16 defgh & Clearwater Russet & 5.3 klmno & 0.02 no \\
\hline Ranger Russet & $21.4 \mathrm{efgh}$ & 0.01 no & Pike & 5.3 klmno & 0.06 hijklmno \\
\hline Kennebec & $21.3 \mathrm{efgh}$ & 0.11 fghijklmn & Premier Russet & $5.3 \mathrm{klmno}$ & 0.02 no \\
\hline Red LaSoda & $21.3 \mathrm{efgh}$ & 0.14 efghijk & Snowden & 5.3 klmno & 0.07 ghijklmno \\
\hline Red Norland & $21.3 \mathrm{efgh}$ & 0.14 efghijkl & Lamoka & $4.0 \mathrm{lmno}$ & 0.02 no \\
\hline Sage Russet & 20.0 efghi & 0.04 klmno & Oneida Gold & 4.0 lmno & 0.02 no \\
\hline Dakota Ruby & 18.6 fghij & 0.15 efghi & Red Thumb & 4.0 lmno & 0.04 lmno \\
\hline French Fingerling & 18.7 fghij & 0.10 fghijklmno & Russian Banana & 4.0 lmno & 0.01 no \\
\hline Ciklamen & 17.3 fghijk & 0.08 ghijklmno & Alturas & $3.0 \mathrm{mno}$ & $0.00 \mathrm{o}$ \\
\hline Alpine Russet & 16.0 ghijkl & 0.15 efghij & Classic Russet & $2.7 \mathrm{mno}$ & 0.02 no \\
\hline Desiree & 14.7 ghijklm & $0.03 \mathrm{mno}$ & Russet Norkotah CO8 & $2.7 \mathrm{mno}$ & $0.00 \mathrm{o}$ \\
\hline Pinnocle & 14.7 ghijklm & 0.11 fghijklmn & Teton Russet & $2.7 \mathrm{mno}$ & 0.01 no \\
\hline Russet Norkotah & 13.3 ghijklmn & $0.03 \mathrm{mno}$ & Bintje & 1.3 no & $0.00 \mathrm{o}$ \\
\hline Yukon Gold & 12.0 ghijklmo & 0.04 lmno & Centennial Russet & 1.3 no & 0.01 no \\
\hline Red Gold & 12.0 ghijklmn & $0.03 \mathrm{mno}$ & Chipeta & 1.3 no & 0.01 no \\
\hline Russet Burbank & 12.0 ghijklmno & 0.13 fghijklm & Dakota Trailblazer & 1.3 no & $0.00 \mathrm{o}$ \\
\hline Superior & 12.0 ghijklmno & 0.05 jklmno & Goldrush & 1.3 no & $0.00 \mathrm{o}$ \\
\hline Huckleberry Gold & 10.7 hijklmno & 0.08 ghijklmn & Mega Chip & 1.3 no & $0.00 \mathrm{o}$ \\
\hline Marcy & 10.7 hijklmno & 0.03 no & Others $^{\mathrm{z}}$ & $0.0 \mathrm{o}$ & $0.00 \mathrm{o}$ \\
\hline Russet Norkotah 278 & 10.7 hijklmno & 0.08 ghijklmno & & & \\
\hline $\mathrm{LSD}_{0.05}$ & & & & 13.1 & 0.1 \\
\hline
\end{tabular}

${ }^{y}$ Means separated by the same letter are not significantly different according to Fisher's protected least significant difference $(\alpha<0.05)$.

${ }^{\mathrm{z}}$ Cultivars (Waneta, Austrian Crescent, Bannock Russet, and Gemstar Russet) with no apparent PMTV-induced tuber necrosis symptoms and not listed in the table. 
storage also influenced the symptom expression in potato cultivars. A 40 day difference between first assessments in 2015 and 2016 trial resulted in 26 additional cultivars affected by PMTV-induced necrosis. More cultivars showing necrosis during 2016 compared with 2015 trial first assessment could also be due to seasonal variability. It was previously reported that within the same field, PMTVinduced tuber incidence differed from year to year (Beuch et al. 2014; Domfeh et al. 2015a; Latvala-Kilby et al. 2009; Nielsen and Molgaard 1997; Santala et al. 2010). In previous studies reported by this research group, seasonal differences in the incidence and severity of powdery scab development and/or PMTV-induced tuber necrosis was attributed to seasonal differences in rainfall and/or irrigation water amounts (Bittara et al. 2016; Carnegie et al. 2010b; Domfeh et al. 2015a). Higher soil moisture levels can lead to higher infection levels of the vector, and therefore, the virus.

Screening results demonstrate that cultivars not affected immediately after harvest may show symptoms over the postharvest storage period. PMTV symptoms are highly influenced by cultivar type (Cooper and Harrison 1973; Domfeh et al. 2015a; Harrison 1974). This could be due to latent PMTV, where symptoms did not manifest after harvest but expressed over the storage duration. These results are similar to previous research (Domfeh et al. 2015a; Kurppa 1989; Nielsen and Molgaard 1997; Rydén et al. 1989). Therefore, understanding the factors causing PMTV-induced necrosis incidence increase under storage conditions may be critical in minimizing quality losses in tubers and attempts have been made to analyze and predict the development of PMTV-induced tuber necrosis over the storage period (Yellareddygari et al. 2017). Further research in the area of tuber necrosis development in storage is warranted.

Results of the cultivar screening experiments demonstrated that an increase in PMTV-induced tuber necrosis is generally correlated with increase in severity, which confirms previous research (Domfeh et al. 2015a, b). Moderate to strong correlation between assessments and between years was observed (based on virus-induced tuber necrosis), an indication that virus-induced tuber necrosis in potato cultivars is consistent and reproducible across years. Due to significant correlations between assessments and between years, we felt it was appropriate to combine the data for cultivar sensitivity rankings.

Cultivar sensitivity rankings for PMTV-induced tuber necrosis show that cultivars Red Endeavor, Viking, Dakota Jewel, and Dark Red Norland had high $(>21 \%)$ incidence in tubers. All of these cultivars are red-skinned market types, suggesting that this potato skin color type may be the most sensitive to PMTV-induced necrosis. It was previously reported that red- and russet-market type cultivars are the most sensitive and insensitive cultivars, respectively (Domfeh et al. 2015a; Yellareddygari et al. 2017). Cultivars Bannock Russet, Gemstar Russet, Lelah, and Waneta have zero PMTV incidence and are categorized as insensitive cultivars. It also appears that most russet-type cultivars are insensitive based on PMTV-induced necrosis data. A previous study found a significant correlation between susceptibility to powdery scab and PMTV-induced tuber necrosis sensitivity (Domfeh et al. 2015a). The most powdery scab susceptible cultivars were also the most sensitive to PMTV necrosis. Russetskinned potato tubers are highly resistant to powdery scab, and highly insensitive to PMTV-induced necrosis (Bittara et al. 2016; Domfeh et al. 2015a).

It is possible that PMTV may exist inside the tubers and not manifest into tuber necrosis. Therefore, this study categorized the cultivars that remained asymptomatic as insensitive rather than resistant. Since cultivars can be symptomless and infected, it has been suggested that they be classified as insensitive rather than resistant (Xenophontos et al. 1998). Future studies should consider using

Table 6. Potato cultivar sensitivity rankings based on Potato mop top virus (PMTV)-induced tuber necrosis incidence values summed for 2015 and 2016 ND trials

\begin{tabular}{|c|c|c|c|c|c|}
\hline Cultivar ${ }^{y}$ & Incidence (\%) & Sensitivity $^{\mathrm{z}}$ & Cultivar $^{y}$ & Incidence (\%) & Sensitivity $^{\mathrm{z}}$ \\
\hline Red Endeavor & 31.8 & $S$ & Russet Norkotah & 3.3 & I \\
\hline Viking & 29.6 & S & Huckleberry Gold & 3.3 & I \\
\hline Dakota Jewel & 24.9 & $S$ & Silverton Russet & 3.2 & I \\
\hline Dark Red Norland & 21.6 & $S$ & Dakota Russet & 3.1 & I \\
\hline Nicolet & 20.3 & S & Clearwater Russet & 2.8 & I \\
\hline Modoc & 18.9 & $S$ & Freedom Russet & 2.7 & I \\
\hline Chieftain & 14.7 & MS & Russet Norkotah $\mathrm{CO} 8$ & 2.7 & I \\
\hline Dakota Pearl & 11.7 & MS & Russet Norkotah 296 & 2.5 & I \\
\hline Dakota Crisp & 10.8 & MS & Red Thumb & 2.5 & I \\
\hline Red Norland & 10.6 & MS & Russet Norkotah CO3 & 2.4 & I \\
\hline Snowden & 10.5 & MS & Premier Russet & 1.9 & I \\
\hline French Fingerling & 10.2 & MS & Umatilla Russet & 1.7 & I \\
\hline Gala & 9.1 & MI & Atlantic & 1.6 & I \\
\hline Yukon Gold & 8.9 & MI & Lamoka & 1.6 & I \\
\hline Kennebec & 8.6 & MI & Alturas & 1.5 & I \\
\hline POR06V12-3 & 8.1 & MI & Oneida Gold & 1.4 & I \\
\hline Red LaSoda & 7.2 & MI & Teton Russet & 1.3 & I \\
\hline Dakota Ruby & 6.3 & MI & Russian Banana & 1.1 & I \\
\hline Ciklamen & 5.8 & MI & Rio Colorado & 0.9 & I \\
\hline Alpine Russet & 5.1 & MI & Dakota Trailblazer & 0.9 & I \\
\hline Desiree & 4.9 & I & Mega Chip & 0.7 & I \\
\hline Snowbird & 4.7 & I & Classic Russet & 0.7 & I \\
\hline Ranger Russet & 4.7 & I & Chipeta & 0.6 & I \\
\hline Pinnacle & 4.6 & I & Bintje & 0.6 & I \\
\hline Marcy & 4.4 & I & Austrian Crescent & 0.4 & I \\
\hline Russet Burbank & 4.3 & I & Centennial Russet & 0.3 & I \\
\hline Russet Norkotah 278 & 4.2 & I & Goldrush & 0.3 & I \\
\hline Red Gold & 3.9 & I & Bannock Russet & 0.0 & I \\
\hline Sage Russet & 3.8 & I & Gemstar Russet & 0.0 & I \\
\hline Superior & 3.7 & I & Lelah & 0.0 & I \\
\hline Pike & 3.5 & I & Waneta & 0.0 & I \\
\hline Colorado Rose & 3.5 & I & .... & $\ldots$ & $\ldots$ \\
\hline
\end{tabular}

${ }^{y}$ Sensitivity ranking of cultivars, Lelah, POR06V12-3, and Rio Colorado were calculated using data from one year.

${ }^{z}$ Sensitivity rankings are insensitive (I), moderately insensitive (MI), moderately sensitive (MS), and sensitive (S). Sensitivity ranking of potato cultivars was calculated by first averaging two assessments per year and then averaging data across two years. 
advanced molecular detection techniques for categorizing cultivars as virus resistant or susceptible. Future improvement in breeding programs for host resistance against PMTV will depend on identifying cultivars that resist infection from PMTV (Domfeh et al. 2015a).

The results presented here demonstrate that there is considerable variation in cultivar sensitivity to tuber necrosis caused by PMTV. PMTV-induced necrosis losses to growers are mainly due to culled potatoes, reductions in contract prices due to exceeding maximum incidence standards, and the cost of fumigation (Brown et al. 2009). Based on cultivar screening results, growers can make decisions on choice of cultivar for disease control under field and storage conditions. These cultivars are readily available and while awaiting a long breeding process, planting insensitive cultivars minimizes tuber quality losses incurred due to either virus. Our results included all the most commonly grown U.S. potato cultivars and inclusion of this information in breeding of resistant cultivars would be valuable.

\section{Acknowledgments}

The authors want to express their gratitude for technical assistance provided by Dean Peterson and Russell Benz.

\section{Literature Cited}

Beuch, U., Persson, P., Edin, E., and Kvarnheden, A. 2014. Necrotic diseases caused by viruses in Swedish potato tubers. Plant Pathol. 63:667-674.

Bittara, F. G., Thompson, A. L., Gudmestad, N. C., and Secor, G. A. 2016. Field evaluation of potato genotypes for resistance to powdery scab on tubers and root gall formation caused by Spongospora subterranea. Am. J. Potato Res. 93:497-508.

Brown, C. R., Mojtahedi, H., Crosslin, J. M., James, S., Charlton, B., Novy, R. G., Love, S. L., Vales, I., and Hamm, P. 2009. Characterization of resistance to corky ringspot disease in potato: A case for resistance to infection by Tobacco rattle virus. Am. J. Potato Res. 86:49-55.

Calvert, E. L. 1968. The reaction of potato varieties to Potato mop-top virus. Rec. Agric. Res. 17:31-40.

Calvert, E. L., and Harrison, B. D. 1966. Potato mop-top, a soil borne virus. Plant Pathol. 15:134-139.

Carnegie, S. F., Cameron, A. M., and McCreath, M. 2010a. Foliar symptoms caused by Potato mop-top virus on potato plants during vegetative propagation in Scotland and their association with tuber yield, spraing and tuber infection. Potato Res. 53:83-93.

Carnegie, S. F., Davey, T., and Saddler, G. S. 2010b. Effect of temperature on the transmission of Potato mop-top virus from seed tuber and by its vector, Spongospora subterranea. Plant Pathol. 59:22-30.

Carnegie, S. F., Davey, T., and Saddler, G. S. 2012. Prevalence and distribution of Potato mop-top virus in Scotland. Plant Pathol. 61:623-631.

Carnegie, S. F., Saddler, G. S., and Peters, J. C. 2009. Cultivar susceptibility to Potato mop-top (PMTV) infection and symptom expression. Asp. Appl. Biol. 94:51-54.

Cooper, J. I., and Harrison, B. D. 1973. Distribution of Potato mop-top virus in Scotland in relation to soil and climate. Plant Pathol. 22:73-78.

David, N., Mallik, I., Crosslin, J. M., and Gudmestad, N. C. 2010. First report of Potato mop-top virus in North Dakota. Plant Dis. 94:1506.

Domfeh, O., Bittara, F. G., and Gudmestad, N. C. 2015a. Sensitivity of potato cultivars to Potato mop-top virus-induced tuber necrosis. Plant Dis. 99:788-796.

Domfeh, O., and Gudmestad, N. C. 2016. Effect of soil moisture management on the development of Potato mop-top virus-induced tuber necrosis. Plant Dis. 100:418-423.

Domfeh, O., Thompson, A. L., and Gudmestad, N. C. 2015b. Sensitivity to tuber necrosis caused by Potato mop-top virus in advanced potato (Solanum tuberosum L.) breeding selections. Am. J. Potato Res. 92:636-647.

Harrison, B. D. 1974. Potato mop-top virus. CMI/AAB Description of Plant Viruses No. 138. Association of Applied Biologists, Wellesbourne, U.K.

Harrison, B. D., and Jones, R. A. C. 1970. Host range and properties of Potato mop-top virus. Ann. Appl. Biol. 65:393-402.
Hu, X. X., Lei, Y., Xiong, X. Y., He, C. Z., Liu, M. Y., and Nie, X. Z. 2013. Identification of Potato mop-top virus (PMTV) in potatoes in China. Can. J. Plant Pathol. 35:402-406.

Jones, R. A. C., and Harrison, B. D. 1969. The behavior of Potato mop-top virus in soil, and evidence for its transmission by Spongospora subterranea (Wallr.) Lagerh. Ann. Appl. Biol. 63:1-17.

Kirk, H. G. 2008. Mop-top virus, relationship to its vector. Am. J. Potato Res. 85: 261-265.

Kurppa, A. 1989. Reaction of potato cultivars to primary and secondary infection by Potato mop-top furovirus and strategies for virus detection. EPPO Bull. 19: 593-598.

Lambert, D. H., Levy, L., Mavrodieva, V. A., Johnson, S. B., Babcock, M. J., and Vayda, M. E. 2003. First Report of Potato mop-top virus on Potato from the United States. Plant Dis. 87:872.

Latvala-Kilby, S., Aura, J. M., Pupola, N., Hannukkala, A., and Valkonen, J. P. T 2009. Detection of Potato mop-top virus in potato tubers and sprouts: combinations of RNA2 and RNA3 variants and incidence of symptomless infections. Phytopathology 99:519-531.

Mallik, I., and Gudmestad, N. C. 2015. First report of Potato mop-top virus causing tuber necrosis in Colorado and New Mexico. Plant Dis. 99:164.

Mumford, R. A., Walsh, K., Barker, I., and Boonham, N. 2000. Detection of Potato mop top virus and Tobacco rattle virus using a multiplex realtime fluorescent reverse-transcription polymerase chain reaction assay. Phytopathology 90: 448-453.

Nakayama, T., Maoka, T., Hataya, T., Shimizu, M., Fuwa, H., Tsuda, S., and Mori, M 2010. Diagnosis of Potato mop-top virus in soil using bait plant bioassay and PCR-microplate hybridization. Am. J. Potato Res. 87:218-225.

Nielsen, S. L., and Engsbro, B. 1992. Susceptibility of potato cultivars to spraing caused by primary infection of Tobacco rattle virus and Potato mop-top virus. Dan. J. Plant Soil Sci. 96:507-516.

Nielsen, S. L., and Molgaard, J. P. 1997. Incidence, appearance and development of Potato mop-top furovirus-induced spraing in potato cultivars and the influence on yield, distribution in Denmark and detection of the virus in tubers by ELISA. Potato Res. 40:101-110.

Rydén, K., Lövgren, L., and Sandgren, M. 1989. Investigations on Potato mop-top furovirus in Sweden. EPPO Bull. 19:579-583.

Sandgren, M. 1995. Potato mop-top virus (PMTV): Distribution in Sweden, development of symptoms during storage and cultivar trials in field and glasshouse. Potato Res. 38:379-389.

Sandgren, M., Plaisted, R. L., Watanabe, K. N., Olsson, S., and Valkonen, J. P. T 2002. Evaluation of some North and South American potato breeding lines for resistance to Potato mop-top virus in Sweden. Am. J. Potato Res. 79:205-210.

Santala, J., Samuilova, O., Hannukkala, A., Latvala, S., Kortemaa, H., Beuch, U., Kvarnheden, A., Persson, P., Topp, K., Ørstad, K., Spetz, C., Nielsen, S. L., Kirk, H. G., Budziszewska, M., Wieczorek, P., Obrępalska-Stęplowska, A., Pospieszny, H., Kryszczuk, A., Sztangret-Wiśniewska, J., Yin, Z., Chrzanowska, M., Zimnoch-Guzowska, E., Jackeviciene, E., Taluntytė, L., Pūpola, N., Mihailova, J., Lielmane, I., Järvekülg, L., Kotkas, K., Rogozina, E., Sozonov, A., Tikhonovich, I., Horn, P., Broer, I., Kuusiene, S., Staniulis, J. Uth, J. G., Adam, G., and Valkonen, J. P. T. 2010. Detection, distribution and control of Potato mop-top virus, a soil-borne virus, in northern Europe. Ann. Appl. Biol. 157:163-178.

Tenorio, J., Franco, Y., Chuquillanqui, C., Owens, R. A., and Salazar, L. F. 2006 Reaction of potato varieties to Potato mop-top virus infection in the Andes. Am. J. Potato Res. 83:423-431.

Whitworth, J. L., and Crosslin, J. M. 2013. Detection of Potato mop-top virus (Furovirus) on potato in southeast Idaho. Plant Dis. 97:149.

Xenophontos, S., Robinson, D. J., Dale, M. F. B., and Brown, D. J. F. 1998 Evidence for persistent, symptomless infection of some potato cultivars with Tobacco rattle virus. Potato Res. 41:255-265.

$\mathrm{Xu}, \mathrm{H} ., \mathrm{DeHaan}, \mathrm{T}$. L., and De Boer, S. H. 2004. Detection and confirmation of Potato mop-top virus in potatoes produced in the United States and Canada. Plant Dis. 88:363-367.

Yellareddygari, S. K. R., Domfeh, O., Bittara, F. G., and Gudmestad, N. C. 2017 Analysis of Potato mop-top virus survival probability in post-harvest storage. Am. J. Potato Res. 94:632-637. 